\title{
The status quo bias and decisions to withdraw life-sustaining treatment
}

\author{
Cite as: CMAJ 2018 March 5;190:E265-7. doi: 10.1503/cmaj.171005
}

t's not uncommon for physicians and surrogate decision-makers to disagree about life-sustaining treatment for incapacitated patients. Several studies show physicians perceive that nonbeneficial treatment is provided quite frequently in their intensive care units. Palda and colleagues, ${ }^{1}$ for example, found that $87 \%$ of physicians believed that futile treatment had been provided in their ICU within the previous year. (The authors in this study equated "futile" with "nonbeneficial," defined as a treatment "that offers no reasonable hope of recovery or improvement, or because the patient is permanently unable to experience any benefit.") Although there is less evidence addressing the prevalence of disagreements or conflicts related specifically to withdrawal of life-sustaining treatment, available evidence shows that such disagreements are not uncommon. Breen and colleagues ${ }^{2}$ interviewed 406 physicians and nurses involved in the care of 102 patients, who reported staff-family conflict in nearly half of the patient cases. In $63 \%$ of those cases causing conflict, the most frequently cited subject of conflict was the decision to withdraw or withhold treatment; $24 \%$ of these conflicts were attributed to family members wanting to continue aggressive treatment against the recommendations of the health care team.

There are myriad reasons why a surrogate decision-maker might disagree with a physician's recommendation to withdraw life-sustaining treatment, including religious beliefs, informational gaps and lack of trust. In some cases, however, these factors may not be present, yet surrogates will still disagree with a recommendation to withdraw treatment. This can be frustrating for clinicians, leaving them uncertain of how to resolve the impasse. One factor that hasn't been studied yet is the role that cognitive biases might play in surrogate decisionmaking regarding withdrawal of lifesustaining treatment. Understanding the role that these biases might play may help improve communication between clinicians and surrogates when these conflicts arise.

\section{Status quo bias}

The classic model of human decisionmaking is the rational choice or "rational actor" model, the view that human beings will choose the option that has the best chance of satisfying their preferences. Making a decision that is inconsistent with one's preferences is therefore considered irrational. However, cognitive psychology research has uncovered a host of psychological phenomena that cause people to make irrational decisions, referred to as "cognitive biases."

One cognitive bias that is particularly worth exploring in the context of surrogate decisions regarding life-sustaining treatment is the status quo bias. This bias, a decision-maker's preference for the current state of affairs, ${ }^{3}$ has been shown to influence decision-making in a wide array of contexts. For example, it has been cited as a mechanism to explain patient inertia (why patients have difficulty changing their behaviour to improve their health), low organ-donation rates, low retirementsaving rates and health plan choices in the United States. ${ }^{3}$ People are psychologically uncomfortable with change and will stick with the current state of affairs, even when it directly conflicts with their preferences.

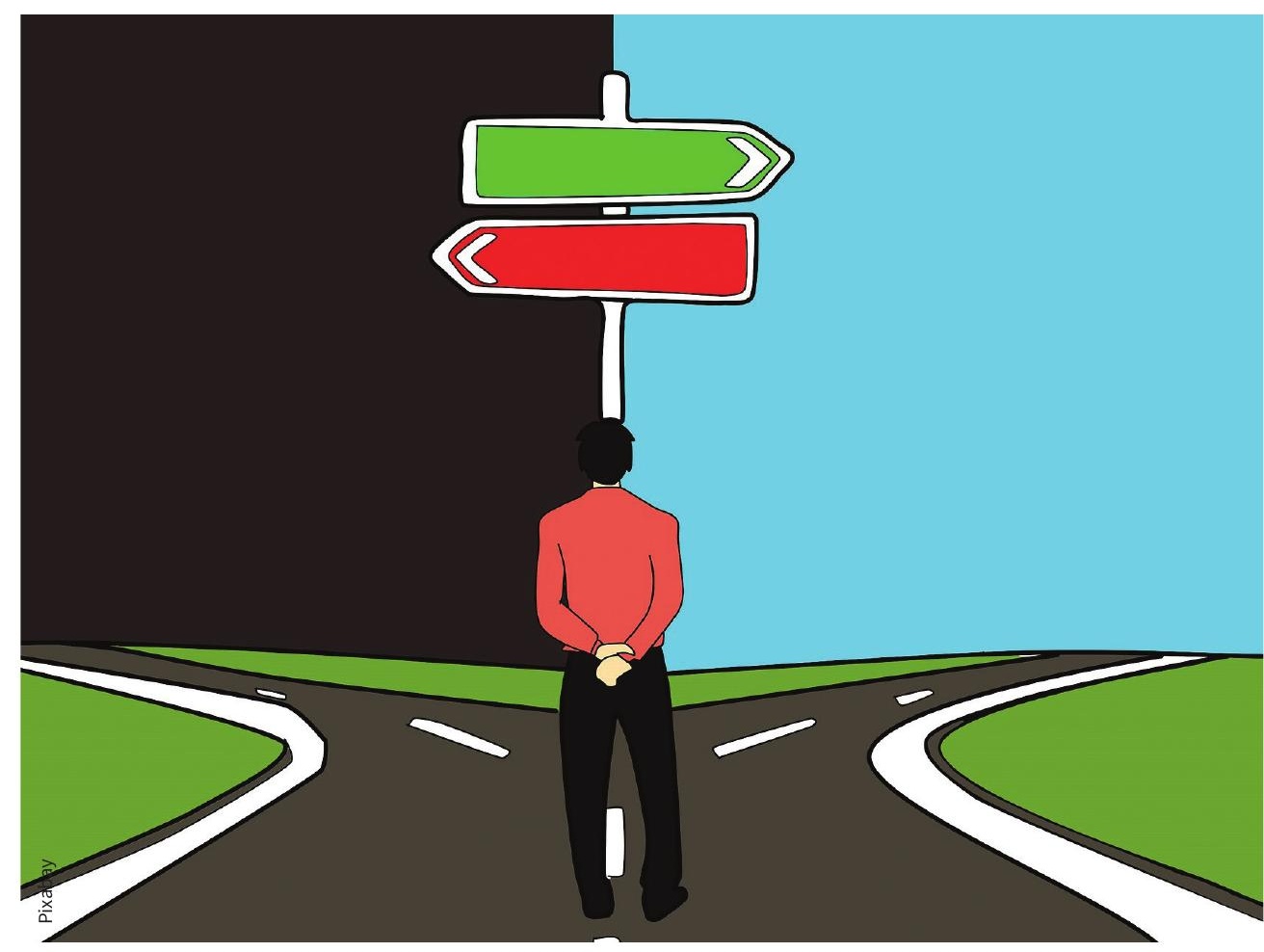


One manifestation of the status quo bias is the default effect: that decisionmakers will tend to stick with the default choice even when it conflicts with their stated preferences. For example, more than one study has shown a powerful default effect among participants completing advance directives, ${ }^{4}$ who were more likely to select treatment options consistent with the default selection than with their own expressed values.

What causes status quo bias? Research has identified two oft-intertwined psychological phenomena as the root cause. One phenomenon is the influence of anticipated regret; the status quo bias functions ated with the illness rather than feel responsible for harm that befalls their children if they make a decision to vaccinate and the child has an adverse outcome. $^{8}$

\section{Life-sustaining treatment}

The decision to consent to withdrawal of life-sustaining treatment may be influenced by the status quo bias. The decision to withdraw is a substantial change in the status quo and may lead the surrogate decision-maker to wonder, "What if we had kept going? Maybe he could have recovered if we gave him more time. Did we make a mistake?"

\section{Cognitive psychology research has uncovered a host of psychological phenomena that cause people to make irrational decisions}

as a regret-minimization strategy..$^{5}$ If we make a decision to change from the status quo and a negative outcome results, we are more likely to imagine that things would have been better if we had just stuck with the status quo - and, therefore, to feel regret associated with our decision. Manetti and colleagues ${ }^{6}$ refer to this as the status quo effect - that decisions to maintain the status quo tend to be regretted less than decisions to change - and describe it as "one of the most robust phenomena in the regret literature."

The second phenomenon is the omission bias: the greater willingness to accept harms that arise from omissions than from actions. ${ }^{7}$ Several authors have suggested that the omission bias stems from a desire to avoid being the direct cause of harm and the perceived greater moral responsibility for being the cause of that harm. ${ }^{3}$ The omission bias has been proposed as an explanation for parental hesitancy and refusal regarding vaccination. These parents choose to put their children at greater risk of harm associ-
The decision to withdraw life-sustaining treatment is also a clear commission; if the surrogate does nothing, the patient will remain on life-sustaining treatment. Even when surrogates accept that the outcomes of both maintaining life-sustaining treatment and withdrawing it are negative, the feeling of greater responsibility for the outcome associated with withdrawing life-sustaining treatment (the patient's death) may motivate some surrogates to opt for maintaining treatment, where they won't feel as responsible for the outcome. Leonhardt, Keller and Pechmann ${ }^{9}$ also suggest that the feeling of responsibility for negative outcomes associated with one's decisions is greater when one's decisions have the potential to harm others as opposed to oneself. Moreover, Anderson ${ }^{3}$ suggests that the perceived responsibility in the eyes of others may also play a role in some situations; if surrogates know that other people attribute less responsibility and wrongdoing to omissions, they could be biased toward omissions so they can avoid blame from others for the bad outcome.
To be clear, this is not meant to suggest that all surrogate disagreements with a physician's recommendation to withdraw life-sustaining treatment can be attributed to the status quo bias, or that such decisions should always be considered irrational. For example, the surrogate may genuinely be carrying out the patient's wishes or acting in accordance with the patient's values and beliefs. In some cases, however, the surrogate may not have any valid reasons for disagreeing with the recommendation, or may be unable or unwilling to consent to withdrawal in accordance with the patient's wishes or values. It is in these cases, where the decision conflicts with the surrogate's preferences to act in the best interests of the patient or to carry out the patient's wishes, and where the decision is therefore irrational, that status quo bias may be the culprit.

\section{Application}

How can clinicians support surrogates to make good decisions on behalf of patients when status quo bias might influence those decisions? One approach is to prevent it from arising in the first place by turning the tables on the default effect. Clinicians can do this before lifesustaining treatment begins (when this is possible) by presenting an alternative to the treatment as the default choice. In cases where the physician judges lifesustaining treatment to be nonbeneficial (e.g., ventilatory support for end-stage pulmonary fibrosis), a comfort measures plan could be presented as the default choice. If the potential benefit is somewhat uncertain, the default choice could be framed as a time-limited trial of treatment followed by withdrawal and comfort measures if the patient does not show signs of improvement. A time-limited trial of treatment has the advantage of setting clear expectations up front and eliciting tacit agreement to withdraw treatment if the trial fails, potentially mitigating the impact of the status quo bias later on.

When life-sustaining treatment has already been started, clinicians can address the influence of the status quo bias by recognizing the signs of the omission bias, empathizing with surrogates who express or imply concerns about withdrawing life-sustaining treatment and then feeling responsible or to 
blame for the patient's death. Physicians can then take steps to mitigate that burden. One approach is to explain to surrogates that it isn't even their decision, it is actually the patient's decision, and their job is to be the voice of the patient and communicate the decision the patient would make.

Another approach is to encourage a single surrogate (e.g., a spouse or lone adult child) to share the decision with others, which both spreads the responsibility and minimizes the risk of being blamed. In extreme cases, it can even be suggested that surrogates consider deferring the decision-making authority to other family members, provided they have sufficient knowledge of the patient's values and beliefs to fulfill the role of surrogate.

A third approach is for the clinician to share responsibility for the decision with the surrogate by taking a more active role in the decision. Instead of laying out the options and asking the surrogate what he or she wants to do, the clinician can provide a recommendation and then ask if the surrogate is okay with proceeding with that recommendation. This has been described by some authors as the notion of "informed assent," and is designed to minimize the emotional and psychological burden associated with being asked to consent to withdrawal. ${ }^{10}$ However, given that recommendations can influence decision-making and can mask value judgments if used in the wrong context, recommendations should be reserved for situations in which they are either based on the patient's known wishes or values, or there is a strong base of evidence and clinical experience supporting the view that treatment would be nonbeneficial. Whichever strategy is chosen, the first step in resolving the issue is recognizing that the status quo bias may be influencing the surrogate's decision-making.

\section{Jonathan Breslin PhD}

Southlake Regional Health Centre, Research \& Innovation, Newmarket, Ont.; Mackenzie Health, Professional Practice, Richmond Hill, Ont.

\section{References}

1. Palda VA, Bowman KW, McLean RF, et al. "Futile" care: Do we provide it? Why? A semistructured, Canada-wide survey of intensive care unit doctors and nurses. J Crit Care 2005;20: 207-13.
2. Breen CM, Abernethy AP, Abbott KH, et al. Conflict associated with decisions to limit lifesustaining treatment in intensive care units. $J$ Gen Intern Med 2001;16:283-9.

3. Anderson CJ. The psychology of doing nothing: forms of decision avoidance result from reason and emotion. Psychol Bull 2003;129:139-67.

4. Halpern SD, Loewenstein G, Volpp KG, et al. Default options in advance directives influence how patients set goals for end-of-life care. Health Aff (Millwood) 2013;32:408-17.

5. Nicolle A, Fleming SM, Bach DR, et al. A regretinduced status quo bias. J Neurosci 2011;31:3320-7.

6. Manetti L, Pierro A, Kruglanski A. Who regrets more after choosing a non-status-quo option? Post decisional regret under need for cognitive closure. J Econ Psychol 2007;28:186-96.

7. Baron J, Ritov I. Omission bias, individual differences, and normality. Organ Behav Hum Decis Process 2004;94:74-85.

8. Ritov I, Baron J. Reluctance to vaccinate: omission bias and ambiguity. J Behav Decis Making 1990;3:263-77.

9. Leonhardt JM, Keller LR, Pechmann C. Avoiding the risk of responsibility by seeking uncertainty: responsibility aversion and preference for indirect agency when choosing for others. J Consum Psychol 2011;21:405-13.

10. Curtis JR, Burt RA. Point: the ethics of unilateral "do not resuscitate" orders: the role of "informed assent”. Chest 2007;132:748-51, discussion 755-6.

This article was solicited and has been peer reviewed. 